3. McIver CJ, Er N, Stevens R, et al. A novel method to enable PCR testing of bacterial isolates in the BacT/ALERT microbial detection system. Pathology 2017; 49: 656-8.

4. Bhatti MM, Boonlayangoor S, Beavis KG, et al. Rapid identification of positive blood cultures by matrix-assisted laser desorption ionizationtime of flight mass spectrometry using prewarmed agar plates. J Clin Microbiol 2014; 52: 4334-8.

5. French K, Evans J, Tanner H, et al. The clinical impact of rapid, direct MALDI-TOF identification of bacteria from positive blood cultures. PLoS One 2016; 11: e0169332.

6. Jakovljev A, Bergh K. Development of a rapid and simplified protocol for direct bacterial identification from positive blood cultures by using matrix assisted laser desorption ionization time-of-flight mass spectrometry. BMC Microbiol 2015; 15: 258-65.

7. La Scola B, Raoult D. Direct identification of bacteria in positive blood culture bottles by matrix-assisted laser desorption ionisation time-offlight mass spectrometry. PLoS One 2009; 4: e8041.

8. Schubert S, Weinert K, Wagner C, et al. Novel, improved sample preparation for rapid direct identification from positive blood cultures using matrix-assisted laser desorption/ionization time-of flight (MALDI-TOF) mass spectrometry. J Mol Diagn 2011; 13: 701-6.

9. Preté PSC, Domingues CC, Meirelles NC, et al. Multiple stages of detergent-erythrocyte membrane interaction - a spin label study. Biochim Biophys Acta 2011; 1808: 164-70.

10. Valentine N, Wunschel S, Wunschel D, et al. Effect of culture conditions on microorganism identification by matrix-assisted laser desorption ionization mass spectrometry. Appl Environ Microbiol 2005; 71: 58-64.

DOI: https://doi.org/10.1016/j.pathol.2018.03.016

\section{A novel splicing mutation in the $A L B$ gene causing analbuminaemia in a Portuguese woman}

Sir,

Homozygous or compound heterozygous variations in the human albumin gene, $A L B$, (GenBank mRNA refseq: NM_000477.6) may cause congenital analbuminaemia (CAA; OMIM \# 616000), a very rare autosomal recessive disorder (prevalence estimated at less than 1 in 1 million), usually characterised by the nearly total absence of albumin (ALB) on conventional or capillary serum protein electrophoresis. $^{1,2}$ Conversely, carriers of the disease have ALB levels close to the lower limit of the normal range, since the normal allele can produce about half the normal ALB amount. ${ }^{1,2}$ CAA is generally thought of as a relatively benign condition in adulthood. The compensatory increase of other serum proteins, which partly can take over the lacking ALB functions, seems to reduce the severity of symptoms. Adult analbuminaemic individuals show few clinical signs of their condition, such as mild oedema, hypotension, fatigue and, mainly in adult females, a peculiar lower-body lipodystrophy and osteoporosis, while the most common biochemical symptoms are gross hyperlipidaemia with hypercholesterolaemia, elevated LDL-cholesterol levels and hypocalcaemia. ${ }^{1,2}$ However, the incidence of CAA and the severity of the phenotype are probably underestimated. In fact, ALB has a crucial role in fetal development, as well as in the peri-natal period and childhood, so that its absence may be life-threatening. ${ }^{3,4}$ In addition, further studies are needed to establish whether CAA can still be regarded as a harmless condition in adults. Normal to high ALB levels have been reported to exert a protective effect against many pathologies, whereas hypoalbuminaemia (ALB $<35 \mathrm{~g} / \mathrm{L}$ ) seems related to the possibility of higher morbidity and mortality. ${ }^{5-7}$ In short, ALB concentration is generally thought of as a remarkably strong prognostic indicator of life expectancy in both sick and seemingly healthy subjects. ${ }^{8}$ Unfortunately, the follow-up of analbuminaemic individuals has been possible only in very few cases so far, making it impossible to establish whether the severe hypercholesterolaemia observed in most patients increases the risk of atherosclerotic complications, or whether their very low ALB level may favour the onset of other diseases.

We describe herein the results of our investigation on an unmarried 40-year-old woman and her mother from Madeira Island (Portugal). The proband presented a history of mild mental retardation, possibly related to birth anoxia, and post-non-Hodgkin lymphoma treatment. She only had a slight oedema of the lower limbs following chemotherapy, which started 5 years previously, but she did not show any lipodystrophy or other symptoms associated with CAA. Her ALB level was low $(<15 \mathrm{~g} / \mathrm{L})$ when measured with a standard clinical chemistry system (AU5400; Beckman Coulter, USA), which uses a photometric bromocresol green dye-binding method, whereas the protein was nearly absent in serum protein capillary electrophoresis (using both SPE and SPE Zoom buffers on the V8 device; Helena Biosciences, UK) (Fig. 1). Also, a nephelometric method (BNProSpec; Siemens Healthineers, Germany) showed the near complete absence of ALB (Table 1). Additionally, agarose gel electrophoresis was performed alongside normal and pathological samples confirming the absence of albumin (Hydragel 4 IF; Sebia, France) (Fig. 1). These data confirm that dye-binding assays inaccurately estimate ALB levels at low concentrations ${ }^{9}$ and that immunochemical methods (nephelometry and turbidimetry) in association with serum protein electrophoresis, routinely performed in clinical chemistry laboratories, represent probably the most accurate method to advance a well-founded suspicion of CAA on a biochemical basis, when the possibility of more common causes of low ALB levels can be ruled out. ${ }^{1,2}$ The total serum protein content of the proband was close to the lower limit of the normal range, because of the compensatory increase of other serum proteins (Table 1). All the lipid fractions were significantly increased (Table 1). The decrease in total serum calcium reflects the corresponding decrease in the fraction normally bound to ALB. Also, the ionised calcium was decreased. Her renal and hepatic functions were normal, and she showed no inflammatory state nor gastrointestinal signs and symptoms, which ruled out the possibility of the common causes of low ALB concentration. The mother, who was 76 years old, showed an ALB level of $33.9 \mathrm{~g} / \mathrm{L}$ (slightly below the lower limit of the normal range). Her electrophoretic profile revealed a slight decrease of the ALB fraction with relative increase of fractions $\alpha-1, \alpha-2, \beta-1$ and $\beta-2$. The total protein content was normal. This behaviour is typical of heterozygous subjects. The father was deceased at the time of our study. The parents of the proband were first degree cousins. The proband had three siblings, one male and two females. All of them were tested for total protein, ALB and protein electrophoresis. Total protein and ALB levels were close to the upper limit of normality, making very unlikely the possibility of a heterozygous condition. Therefore, we decided to exclude them from the genetic study. 
A
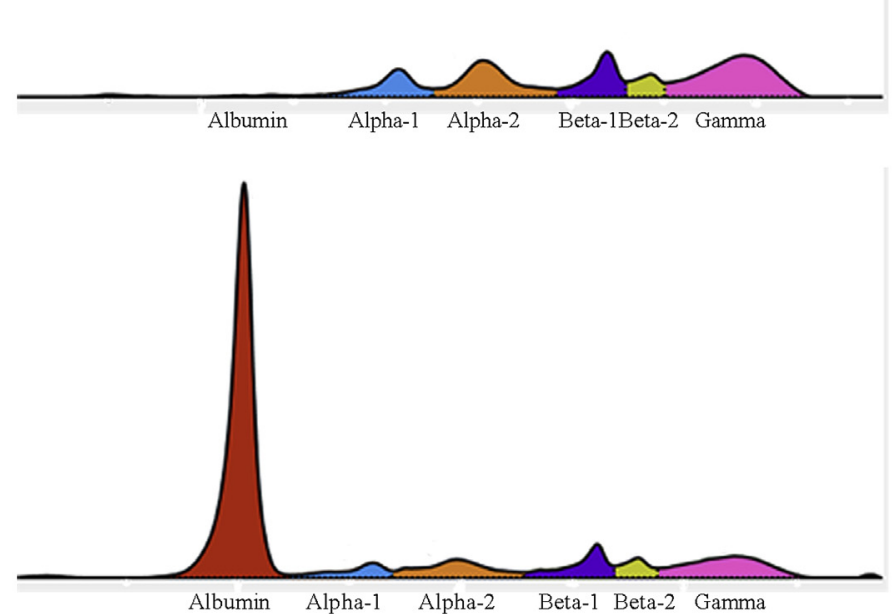

B

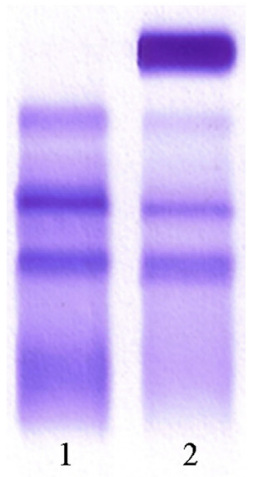

Fig. 1 Serum protein electrophoresis of the analbuminaemic patient. (A) Capillary electrophoresis: upper part, patient; lower part, control. (B) Agarose gel electrophoresis: lane 1 , patient; lane 2, control. The results show the almost complete absence of serum albumin, and compensatory increases in alpha-1, alpha-2, beta and gamma fractions.

To confirm in our patient the diagnosis of CAA, based on the above-mentioned clinical and biochemical findings, a mutation analysis of the $A L B$ was carried out following the principles outlined in the Declaration of Helsinki. After we obtained informed consent, genomic DNA was extracted from whole blood of the proband, her mother, and two unrelated healthy volunteers as a control. Fourteen genomic

Table 1 Clinical laboratory values of the analbuminaemic patient

\begin{tabular}{|c|c|c|c|}
\hline Analyte & Result & Units & $\begin{array}{c}\text { Reference } \\
\text { interval }\end{array}$ \\
\hline Serum albumin (nephelometric) & $<0.341$ & $\mathrm{~g} / \mathrm{L}$ & $35-52$ \\
\hline Serum albumin (colorimetric) & $<15$ & $\mathrm{~g} / \mathrm{L}$ & $35-48$ \\
\hline Urine albumin & $<0.07$ & $\mathrm{~g} / \mathrm{L}$ & $<0.2$ \\
\hline Serum total protein & 58.4 & $\mathrm{~g} / \mathrm{L}$ & $60-80$ \\
\hline Urine total protein & 0.05 & $\mathrm{~g} / \mathrm{L}$ & $<0.1$ \\
\hline Albumin $^{\mathrm{a}}$ & 2.8 & $\mathrm{~g} / \mathrm{L}$ & $38-49$ \\
\hline Alpha-1-globulins ${ }^{\mathrm{a}}$ & 7.3 & $\mathrm{~g} / \mathrm{L}$ & $1.8-3.5$ \\
\hline Alpha-2-globulins ${ }^{\mathrm{a}}$ & 13.7 & $\mathrm{~g} / \mathrm{L}$ & $3.4-7.4$ \\
\hline Beta-1-globulins ${ }^{\mathrm{a}}$ & 9.0 & $\mathrm{~g} / \mathrm{L}$ & $3.8-6.4$ \\
\hline Beta-2-globulins ${ }^{\mathrm{a}}$ & 4.8 & $\mathrm{~g} / \mathrm{L}$ & $1.7-5.0$ \\
\hline Gamma-globulins $^{\mathrm{a}}$ & 20.8 & $\mathrm{~g} / \mathrm{L}$ & $6.8-15$ \\
\hline Ratio albumin/globulins & 0.05 & ratio & $0.99-1.81$ \\
\hline $\operatorname{IgA}$ & 2.82 & $\mathrm{~g} / \mathrm{L}$ & $0.7-4.0$ \\
\hline $\operatorname{IgG}$ & $\mathbf{1 7 . 5}$ & $\mathrm{g} / \mathrm{L}$ & $7-16$ \\
\hline $\operatorname{IgM}$ & 1.81 & $\mathrm{~g} / \mathrm{L}$ & $0.4-2.3$ \\
\hline $\operatorname{IgE}$ & 710 & $\mu \mathrm{g} / \mathrm{L}$ & $<288$ \\
\hline $\operatorname{IgG} 1$ & 12.6 & $\mathrm{~g} / \mathrm{L}$ & $4.05-10.11$ \\
\hline IgG2 & 4.44 & $\mathrm{~g} / \mathrm{L}$ & $1.69-7.86$ \\
\hline $\operatorname{IgG3}$ & 1.2 & $\mathrm{~g} / \mathrm{L}$ & $0.11-0.85$ \\
\hline IgG4 & 0.397 & $\mathrm{~g} / \mathrm{L}$ & $0.03-2.01$ \\
\hline Transferrin & 4.226 & $\mathrm{~g} / \mathrm{L}$ & $2.0-3.6$ \\
\hline Alpha-1-antitrypsin & 3.19 & $\mathrm{~g} / \mathrm{L}$ & $0.9-2.0$ \\
\hline Caeruloplasmin & 0.512 & $\mathrm{~g} / \mathrm{L}$ & $0.22-0.58$ \\
\hline Haptoglobulin & 0.97 & $\mathrm{~g} / \mathrm{L}$ & $0.3-2.0$ \\
\hline $\mathrm{C} 3$ & 2.01 & $\mathrm{~g} / \mathrm{L}$ & $0.9-1.8$ \\
\hline $\mathrm{C} 4$ & 0.399 & $\mathrm{~g} / \mathrm{L}$ & $0.1-0.4$ \\
\hline Total cholesterol & 11.86 & $\mathrm{mmol} / \mathrm{L}$ & $<5.18$ \\
\hline HDL cholesterol & 4.7 & $\mathrm{mmol} / \mathrm{L}$ & $1.03-1.55$ \\
\hline LDL cholesterol & 6.6 & $\mathrm{mmol} / \mathrm{L}$ & $<4.14$ \\
\hline Triglycerides & 2.38 & $\mathrm{mmol} / \mathrm{L}$ & $<1.8$ \\
\hline Serum calcium & 1.92 & $\mathrm{mmol} / \mathrm{L}$ & $2.23-2.58$ \\
\hline Serum ionised calcium & 0.76 & $\mathrm{mmol} / \mathrm{L}$ & $1.16-1.32$ \\
\hline
\end{tabular}

Results falling outside the reference interval are in bold.

${ }^{a}$ Determined by capillary electrophoresis. fragments of the $A L B$, encompassing the coding exons and their intron-exon junctions, were polymerase chain reaction (PCR) amplified using specific primer pairs as described by Watkins et al., ${ }^{3}$ and then studied by heteroduplex and singlestrand conformational polymorphism analysis. However, in this case this strategy did not allow an unambiguous identification of the mutation site and therefore all the fragments were submitted to direct DNA sequencing. This revealed that the only molecular defect was located in the $356 \mathrm{bp}$ long region amplified by using PCR primers A05A and A06A encompassing exon 3 and its intron-exon junctions. ${ }^{3,10}$ The patient was homozygous for a $A>G$ transition at nucleotide c.138-2, the second last base of intron 2, and the mother was heterozygous for the wild type and mutated alleles, as the sequencing electropherograms showed the presence of two superimposed peaks at that position, representing the normal (A) and the variant (G) base (Fig. 2). These results, confirming the inheritance of the trait, allowed the identification of a novel c.138-2 A $>$ G variant, for which we suggest the name Madeira, as the cause of CAA in our patient. The c.138-2 A $>$ G variant is described as 'likely pathogenic' according to ACMG guidelines. In fact, it is located within \pm 2 of canonical splice site (rule PVS1) and the allele was not found in GnomAD and ExAC Exome Aggregation Consortium databases (rule PM2). ${ }^{10}$ All 14 junctions present in the ALB conform with the GT and AG consensus sequences present at the $5^{\prime}$ and $3^{\prime}$ splice sites, respectively. ${ }^{11}$ The Madeira variant destroys the invariant AG dinucleotide sequence at the acceptor splice site of intron 2, and it is well known that intronic alterations located in consensus splicing regions lead to aberrant transcript(s), either lacking one or more exons, or even part of them, or retaining intronic sequences. ${ }^{12}$ We could not establish the effects of this splicing mutation at the mRNA and, consequently, at the protein level, but no evidence has been so far reported for the presence of aberrant truncated ALB variants in the serum of analbuminaemic individuals. ${ }^{1,2}$

The Madeira variant is the 25th different defect identified as a cause of CAA among the 47 cases so far characterised at the molecular level: ten mutations affecting splicing, seven nonsense mutations, six frame-shift/deletions, one frame- 


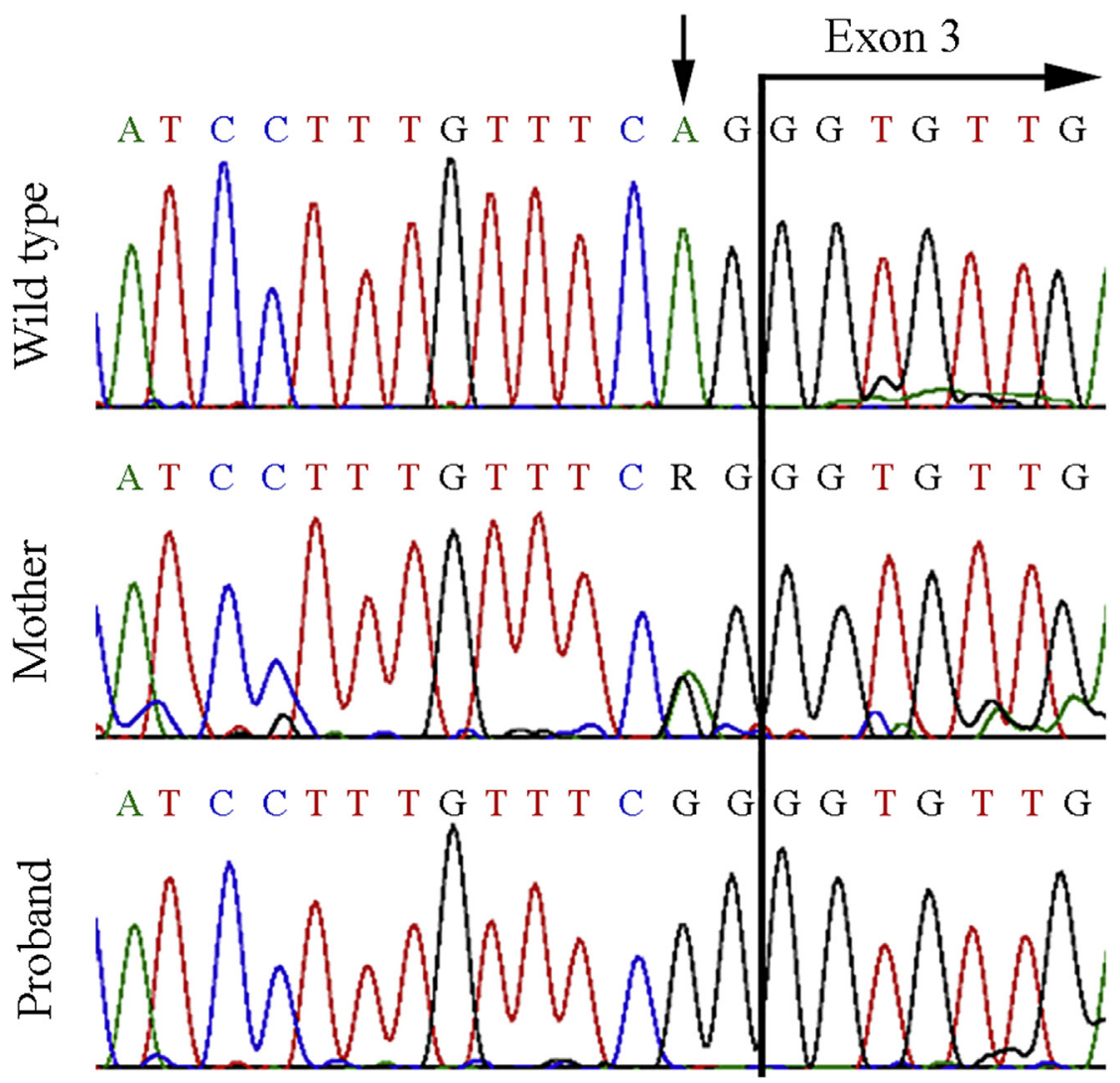

Fig. 2 Genomic DNA sequence electropherograms of the members of the Portuguese family. The region encompassing exon 3 and the intron2-exon3 and exon3intron 3 junctions was amplified using PCR primers A05A and A06A and then submitted to direct DNA sequencing. The arrow indicates the A $>\mathrm{G}$ transition at nucleotide c.138-2, the second last base of intron 2. The patient is homozygous for this mutation and the mother heterozygous for the wild type and mutated alleles, as seen by the presence of two superimposed peaks.

shift/insertion and one mutation in the start codon. ${ }^{2,13}$ Of the molecular defects affecting splicing, the Madeira variant is the second identified at an acceptor splice site, whereas five are at donor splice sites, suggesting that the latter are more prone to mutations. ${ }^{2}$ The finding that the CAA causing variants are located in nine different exons and in seven different introns indicates that CAA is the result of widely scattered and randomly occurring different molecular defects. Whereas the Madeira variant, as most of the other causative defects, is so far unique, two mutations have been identified in apparently unrelated and geographically distant families: the two base deletion c.228_229delAT (analbuminaemia Kayseri), which is by far the most frequent cause of the trait, accounting for 13 of the cases characterised at the molecular level, and the splicing defect c. $1289+1 \mathrm{G}>\mathrm{A}$ of analbuminaemia Guimarães, identified in four cases. ${ }^{2,13}$ These defects seem to be located in mutational hot spots of the $A L B$. The cumulative frequency of the CAA causing variants reported in the large population sequencing project, ExAC, is 8.3 in 100,000 . It is worth noting that Kayseri has the highest allele frequency at the heterozygous state in the average normal population $(5.8$ in 100,000$) .{ }^{10}$ In contrast, the cumulative frequency of the $A L B$ variants known to cause alloalbuminaemia, a benign condition characterised by the presence of a circulating variant of ALB, is significantly higher: 2.17 in $1000{ }^{10}$ The very low frequency of the CAA causing variants supports the hypothesis that the lack of ALB may be lethal during the fetal life or in the perinatal period.
The data here reported allowed us to identify the 25th different variant, a c.138-2 $\mathrm{A}>\mathrm{G}$ defect in intron 2 , as the cause of CAA in our patient. The results expand the mutation spectrum of $A L B$ underlying this condition and contribute to shed light on the patho-physiology and on the molecular genetics of CAA.

Conflicts of interest and sources of funding: The authors state that there are no conflicts of interest to disclose.

Gianluca Caridi ${ }^{1}$, Ilidio B. R. de Abreu ${ }^{2}$, José A. Alves ${ }^{2}$, Francesca Lugani ${ }^{1}$, Monica Campagnoli ${ }^{3}$, Monica Galliano ${ }^{3}$, Lorenzo Minchiotti ${ }^{3}$

${ }^{1}$ Laboratory of Molecular Nephrology, Istituto Giannina Gaslini, IRCCS, Genoa, Italy; ${ }^{2}$ Hospital Dr Nélio Mendonça, Clinical Pathology Department, Serviço de Saúde da Região Autónoma da Madeira EPE, Funchal, Portugal; ${ }^{3}$ Department of Molecular Medicine, University of Pavia, Pavia, Italy

Contact Lorenzo Minchiotti.

E-mail: loremin@unipv.it

1. Minchiotti L, Galliano M, Caridi G, Kragh-Hansen U, Peters Jr T. Congenital analbuminaemia: molecular defects and biochemical and clinical aspects. Biochim Biophys Acta 2013; 1830: 5494-502.

2. The Albumin Website. Cited Dec 2017. http://www.albumin.org.

3. Watkins S, Madison J, Galliano M, Minchiotti L, Putnam FW. A nucleotide insertion and frameshift cause analbuminemia in an Italian family. Proc Natl Acad Sci USA 1994; 91: 2275-9. 
4. Toye JM, Lemire EG, Baerg KL. Perinatal and childhood morbidity and mor tality in congenital analbuminemia. Paediatr Child Health 2012; 17: 20-3.

5. Ha CE, Masaki KH, Petrovitch H, et al. Human serum albumin levels and cardiovascular risk factors in elderly Japanese-American men: the Honolulu Heart Program. Hawaii Med J 2007; 66: 150-2.

6. Ashinnia F, Pennathur S. Association of hypoalbuminemia with osteoporosis: analysis of the National Health and Nutrition Examination Survey. J Clin Endocrinol Metab 2016; 101: 2468-74.

7. Sunghye K, McClave SA, Martindale RG, Miller KR, Hurt RT. Hypoalbuminemia and clinical outcomes: what is the mechanism behind the relationship? Am Surg 2017; 83: 1220-7.

8. Levitt DG, Levitt MD. Human serum albumin homeostasis: a new look at the roles of synthesis, catabolism, renal and gastrointestinal excretion, and the clinical value of serum albumin. Int J Gen Med 2016; 9: 229-55.

9. Lyon AW, Meinert P, Bruce GA, Laxdal VA, Salkie ML. Influence of methodology on the detection and diagnosis of congenital analbuminaemia. Clin Chem 1998; 44: 2365-7.

10. Exome Aggregation Consortium (ExAC). ExAC Browser (Beta). Cited Dec 2017. http://exac.broadinstitute.ors

11. Minghetti PP, Ruffner DE, Kuang WJ, et al. Molecular structure of the human albumin gene is revealed by nucleotide sequence within q11-22 of chromosome 4. J Biol Chem 1986; 261: 6747-57.

12. Ward AJ, CooperTA. The pathobiology of splicing. J Pathol 2010; 220: 152-63.

13. Caridi G, Maout A, Artan R, et al. Congenital analbuminemia in unrelated Algerian and Turkish families is caused by the same molecular defect in the albumin gene. Ann Lab Med 2018; 38: 185-8.

DOI: https://doi.org/10.1016/j.pathol.2018.03.015

\section{HPV positive oesophageal squamous cell carcinoma presenting as a metastasis to the thyroid masquerading as a primary malignancy, diagnosed by cytology}

Sir,

Intra-thyroid metastasis (ITM) is uncommon despite the thyroid gland being a highly vascular organ, second only to adrenal glands in relative vascular perfusion. The overall incidence of ITM ranges from $0.6 \%$ to $5.7 \%$ with metastatic squamous cell carcinoma (SCC) an even rarer entity. ${ }^{1} \mathrm{Au}-$ topsy series report higher rates, ranging from $1.25 \%$ to $24 \%$ but most of these cases are not detected clinically., 2,3

The oncogenic role of human papillomaviruses (HPV) in cervical cancer is well known but despite multiple metaanalyses their effect on esophageal SCC remains controversial. There are significant worldwide geographical differences in the overall incidence of HPV related oesophageal SCC, varying from $11.7 \%$ to $38.9 \%$, and Australia is categorised as a low risk country having reported rates of 2.5 per $100,000 .{ }^{4}$ Herein we present the first case of metastatic HPV positive SCC of the thyroid originating in the oesophagus of a young male.

A 44-year-old man presented with 6 week history of worsening odynophagia and dysphagia. A computed tomography scan of the head and neck showed trachea compression by a $50 \mathrm{~mm}$ mass from the lower pole of the left thyroid gland without localised lymphadenopathy. A primary thyroid malignancy was suspected and urgent surgery was planned. An ultrasound guided fine needle aspiration (FNA) was performed and five air-dried smears and a cell block were received. Smears revealed malignant epithelial cells, some spindly, occurring singly and in clusters, showing dense cytoplasm suggestive of squamous differentiation (Fig. 1A). Immunohistochemistry (IHC) performed on a cell block showed positive staining for CK5/6, p63, p40 and p16 confirming the squamous nature (Fig. 1B).

The possibilities considered were primary thyroid SCC, anaplastic thyroid carcinoma with a squamous component or metastatic SCC. Given the age of the patient and rarity of primary anaplastic and squamous cell carcinoma, the likelihood of metastasis needed exclusion. These findings were discussed with the clinical team and immediate surgery was postponed until further investigation.

Polymerase chain reaction (PCR) HPV DNA testing was performed as the topographic site is in close proximity to the oropharynx that is commonly known to be associated with high risk HPV infections and primary thyroid carcinomas have never been shown to be associated with HPV infection. The results were positive for high risk HPV-16 genotype and the finding was highly suggestive of metastatic SCC of the head and neck region.

The patient proceeded to have an urgent oesophagoscopy that showed a $40 \mathrm{~mm}$ exophytic upper oesophageal tumour at $15-20 \mathrm{~cm}$. Histology of the mass biopsy showed a similar morphology (Fig. 2A), immunophenotype, and HPV-16 genotype (Fig. 2B) to the thyroid deposit, confirming the diagnosis of a primary moderately differentiated oesophageal SCC with metastases to thyroid. Staging imaging demonstrated no other distant metastases.

The patient was referred to the otolaryngology multidisciplinary team (MDT) and was commenced on chemoradiotherapy. The management was complicated by tracheal stenosis and a stent was inserted. Follow up positron emission tomography scan showed complete metabolic response of both the upper oesophageal mass and the ITM. Subsequent bronchial brushings, tracheal/bronchial washings, subglottic and lower trachea biopsies were all negative for malignancy. The patient remains under close surveillance and further follow up 18 months post-diagnosis revealed no disease recurrence.

ITM is relatively uncommon and can often masquerade as a primary thyroid neoplasm. The distinction is significant as they have different management pathways. The potential long latency period for the appearance of metastases makes it seem unrelated to the primary malignancy many years previously, representing a diagnostic pitfall for the unsuspecting pathologist.

ITM can originate from any organ system with commonly reported sites including lung, kidney, breast, skin and oesophagus. In cases with poorly differentiated disease the site of origin may not be identifiable. Autopsy series have reported lung to be the most common primary origin of ITM, whereas clinical series have shown renal cell carcinoma to be more common. ${ }^{5}$ It is thought that this difference may be attributed to the variable clinical presentation, nature of disease and differing management guidelines. ${ }^{3,5}$

The appearance of ITM can be the first presentation of metastases or can occur simultaneously with other metastases. The lag time between the diagnosis of a primary malignancy and the appearance of ITM can be years (range 1 month -26 years) and often signifies poor prognosis with an average survival of 12 months. ${ }^{3,6-8}$ The short survival time may reflect the advanced stage of disease progression at diagnosis or could be due to the aggressive nature of disease. Oesophageal carcinoma with ITM often represents a preterminal event.

Cases with ITM are commonly identified via three different scenarios: (1) patients can present with thyroid 